九州大学学術情報リポジトリ

Kyushu University Institutional Repository

\title{
BOUNDED RISK POINT ESTIMATION OF THE SCALE PARAMETER OF A NEGATIVE EXPONENTIAL DISTRIBUTION
}

\section{Isogai, Eiichi}

Department of Mathematics, Ni igata University

Saito, Kazumasa

Graduate School of Science and Technology, Niigata University

Uno, Chikara

Department of Mathematics, Akita University

https://doi.org/10.5109/13479

出版情報: Bulletin of informatics and cybernetics. 31 (1), pp.35-46，1999-03. Research Association of Statistical Sciences

バージョン :

権利関係 : 


\title{
BOUNDED RISK POINT ESTIMATION OF THE SCALE PARAMETER OF A NEGATIVE EXPONENTIAL DISTRIBUTION
}

\author{
By \\ Eiichi IsogaI*, Kazumasa $\mathrm{SAIT}^{\dagger}$ and Chikara $\mathrm{UNO}^{\ddagger}$

\begin{abstract}
We consider the problem of bounded risk point estimation for the scale parameter of a negative exponential distribution under a certain loss function. In this paper we propose a stopping rule and two sequential estimators for the scale parameter. The asymptotic expansions of the risk associated with the sequential estimators are obtained.
\end{abstract}

Keywords and Phrases: Bounded risk ; negative exponential distribution ; asymptotic expansion ; unifom integrability.

AMS (1991) subject classification: Primary $62 \mathrm{~L} 12$.

\section{Introduction}

Let $X_{1}, X_{2}, \ldots$ be independent and identically distributed (i.i.d)random variables with the probability density function (pdf)

$$
f_{\mu, \sigma}(x)=\sigma^{-1} \exp \{-(x-\mu) / \sigma\} I(x \geq \mu),
$$

where $-\infty<\mu<\infty$ and $0<\sigma<\infty$ are two unknown parameters, and $I(A)$ denotes the indicator function of $A$. This paper deals with the problem of bounded risk point estimation for the scale parameter $\sigma$. Suppose that $\delta_{n}=\delta_{n}\left(X_{1}, \ldots, X_{n}\right)$ is an estimator of $\sigma$ based on a sample $X_{1}, \ldots, X_{n}$ of size $n$ and $R\left(\delta_{n}\right)$ is a risk associated with $\delta_{n}$. We wish to estimate $\sigma$ by use of the smallest sample size satisfying $R\left(\delta_{n}\right) \leq w$ with a preassigned constant $w>0$. In this paper, we give an estimator $\delta_{n}$ and its risk $R\left(\delta_{n}\right)$, and consider this problem. Then for small $w$ we can find an asymptotically optimal sample size. However, the optimal sample size contains the unknown scale parameter. Therefore, we will propose a stopping rule and give a natural sequential estimator of $\sigma$. Furthermore, we will propose another sequential estimator the risk of which is smaller than that of the natural sequential estimator. Sequential and multistage estimation

\footnotetext{
* Department of Mathematics, Niigata University, Niigata 950-2181, Japan

$\dagger$ Graduate School of Science and Technology, Niigata University, Niigata 950-2181, Japan

† Department of Mathematics, Akita University, Akita 010-8502, Japan
} 
problems for $\sigma$ were reviewed in Mukhopadhyay (1988) and Ghosh, Mukhopadhyay and Sen (1997) (Section 6.6), for example.

In Section 2 we formulate the problem of bounded risk point estimation for $\sigma$ and provide results. Section 3 gives their proofs.

\section{Formulation and results}

In this section we consider the problem of bounded risk point estimation for the scale parameter $\sigma$. Let $X_{1}, X_{2}, \ldots$ be i.i.d random variables with pdf $f_{\mu, \sigma}$ given by (1.1). Set

$$
T_{n}=\min \left(X_{1}, \ldots, X_{n}\right) \text { and } \hat{\sigma}_{n}=(n-1)^{-1} \sum_{i=1}^{n}\left(X_{i}-T_{n}\right) \text { for } n \geq 2 .
$$

In the paper we use the estimator $\hat{\sigma}_{n}$ for $\sigma$. Define the loss function by

$$
L\left(\hat{\sigma}_{n}\right)=\left(\hat{\sigma}_{n}-\sigma\right)^{2} / \sigma .
$$

The risk associated with $\hat{\sigma}_{n}$ is given by

$$
R\left(\hat{\sigma}_{n}\right)=E\left\{L\left(\hat{\sigma}_{n}\right)\right\}=(n-1)^{-1} \sigma .
$$

This weighted loss function is appropriate when there is a possibility that $\sigma$ may be close to 0 , so that small absolute error is too weak a requirement to place on the estimate of $\sigma$. Similary the above loss function makes sense if $\sigma$ may be very large since in that case the requirment of small absolute error may be too stringent. Note that this loss function has the same dimension as observations. Let $w>0$ be a preassigned number. Then we wish to estimate $\sigma$ by $\hat{\sigma}_{n}$ under the above loss function and find the smallest sample size which will satisfy that the risk $R\left(\hat{\sigma}_{n}\right)$ is not greater than $w$. From (2.1)

$$
R\left(\hat{\sigma}_{n}\right) \leq w
$$

is equivalent to

$$
n \geq(\sigma / w)+1
$$

Let

$$
n^{*}=\sigma / w \text { and } n_{0}=n^{*}+1 .
$$

For simplicity, $n^{*}$ is assumed to be an integer. Then $n_{0}$ is the optimal fixed sample size in the sense that $n_{0}$ is the smallest sample size which satisfies $R\left(\hat{\sigma}_{n}\right) \leq w$. Unfortunately, $n_{0}$ contains the unknown parameter $\sigma$ and there is no fixed sample size procedure which will satisfy that the risk $R\left(\hat{\sigma}_{n_{0}}\right)$ with the optimal sample size $n_{0}$ is not greater than $w$. Thus, taking account of $(2.2)$ we propose the following stopping rule by which the sampling is stopped :

$$
N=N_{w}=\inf \left\{n \geq m: \sum_{i=1}^{n}\left(X_{i}-T_{n}\right) \leq w(n-1)^{2} l(n-1)\right\},
$$


where $m \geq 2$ is a starting sample size and $l(x)$ is a given continuous function on $(0, \infty)$ satisfying that $l(x)>0$ on $(0, \infty)$ and

$$
l(x)=1+l_{0} x^{-1}+o\left(x^{-1}\right) \text { as } x \rightarrow \infty
$$

with a constant $l_{0}$. Since $P\{N<\infty\}=1$ for each $w>0$, we estimate $\sigma$ by $\hat{\sigma}_{N}$. Then the risk associated with $\hat{\sigma}_{N}$ is given by $R(\hat{\sigma}, N)=E\left\{\left(\hat{\sigma}_{N}-\sigma\right)^{2} / \sigma\right\}$ with $\hat{\sigma}=\left\{\hat{\sigma}_{n} ; n \geq\right.$ $1\}$.

We shall first give asymptotic expansions of the expected sample size $E(N)$ and $R(\hat{\sigma}, N) / w$.

THEOREM 2.1. The following results hold.

(i) For $m \geq 2$,

$$
E(N)=n^{*}+\nu-l_{0}+o(1) \text { as } w \rightarrow 0
$$

(ii) For $m \geq 6$,

$$
R(\hat{\sigma}, N) / w=1-\left(\nu-l_{0}-4\right) / n^{*}+o\left(1 / n^{*}\right) \text { as } w \rightarrow 0,
$$

where $\nu$ is the constant given in Lemma 3.1 (iii) and approximately 0.747 .

REMARK 2.1. (i) If $v \equiv \nu-l_{0}-4>0$, then $R(\hat{\sigma}, N)<w$ for $w>0$ sufficiently small. (ii) The larger the value of $v$ is, the smaller $R(\hat{\sigma}, N) / w$ is, although the expected sample size becomes larger.

The estimator $\hat{\sigma}_{n}$ is unbiased, but we do not know whether or not $\hat{\sigma}_{N}$ is unbiased. The following proposition provides the bias of $\hat{\sigma}_{N}$.

PROPOSITION 2.1. For $m \geq 4$,

$$
E\left(\hat{\sigma}_{N}\right)-\sigma=-w+o(w) \text { as } w \rightarrow 0 .
$$

According to this proposition, we consider the bias-corrected estimators

$$
\hat{\sigma}^{*}=\left\{\hat{\sigma}_{n}^{*} ; n \geq 1\right\} \text { and } \hat{\sigma}_{n}^{*}=\hat{\sigma}_{n}+w .
$$

Then, the asymptotic expansion of $R\left(\hat{\sigma}^{*}, N\right) / w$ associated with the bias-corrected estimator $\hat{\sigma}_{N}^{*}$ is given by the following theorem.

THEOREM 2.2. For $m \geq 6$,

$$
R\left(\hat{\sigma}^{*}, N\right) / w=1-\left(\nu-l_{0}-3\right) / n^{*}+o\left(1 / n^{*}\right) \text { as } w \rightarrow 0
$$


We compare now the sequential procedure $\left(\hat{\sigma}^{*}, N\right)$ with $(\hat{\sigma}, N)$ from the viewpoint of second order asymptotic relative efficiency. From Theorems 2.1 and 2.2 we have

$$
n^{*}\left\{R(\hat{\sigma}, N)-R\left(\hat{\sigma}^{*}, N\right)\right\} / w=v^{*}-v+o(1) \text { as } w \rightarrow 0,
$$

where $v=\nu-l_{0}-4$ and $v^{*}=\nu-l_{0}-3$. Choose $l_{0}<\nu-4$ so that $v$ and $v^{*}$ are positive. Then both $R(\hat{\sigma}, N) / w$ and $R\left(\hat{\sigma}^{*}, N\right) / w$ are less than 1 for $w>0$ sufficiently small and $v^{*}-v=1$. The value $v^{*}-v>0$ means that $n^{*}\left\{1-R\left(\hat{\sigma}^{*}, N\right) / w\right\}$ is asymptotically $v^{*}-v$ greater than $n^{*}\{1-R(\hat{\sigma}, N) / w\}$. Therefore, the procedure $\left(\hat{\sigma}^{*}, N\right)$ is asymptotically more efficient than $(\hat{\sigma}, N)$ in the above sense.

REMARK 2.2. For example, let us take $l(x)=\left(1-2 x^{-1}\right)^{2}+x^{-2}$ in (2.5). Then $l_{0}=-4<\nu-4$, and $v=\nu>0$ and $v^{*}=\nu+1>0$. Thus, as $w \rightarrow 0$

$$
R(\hat{\sigma}, N) / w=1-\nu / n^{*}+o\left(1 / n^{*}\right)
$$

and

$$
R\left(\hat{\sigma}^{*}, N\right) / w=1-(\nu+1) / n^{*}+o\left(1 / n^{*}\right) .
$$

In this case, we have for $w$ sufficiently small

$$
R(\hat{\sigma}, N)<w \text { and } R\left(\hat{\sigma}^{*}, N\right)<w .
$$

Therefore the condition on the risk is asymptotically satisfied.

\section{Proofs}

In this section we shall give the proofs of the results in Section 2. Let

$$
Y_{i n}=(n-i+1)\left(X_{n(i)}-X_{n(i-1)}\right) \text { for } i=2, \cdots, n,
$$

where $X_{n(1)} \leq X_{n(2)} \leq \cdots \leq X_{n(n)}$ are the order statistics of $X_{1}, \ldots, X_{n}$. Then $Y_{2 n}, \ldots, Y_{n n}$ are i.i.d random variables with pdf $f_{0, \sigma}$ and $\hat{\sigma}_{n}=(n-1)^{-1} \sum_{i=2}^{n} Y_{i n}$. Let $W_{1}, W_{2}, \ldots$, be a sequence of i.i.d random variables with pdf $f_{0,1}$ and put

$$
S_{n}=\sum_{i=1}^{n} W_{i}, \quad \bar{W}_{n}=S_{n} / n \text { and } Z_{n}=\sigma W_{n}
$$

Define

$$
Q=Q_{w}=\inf \left\{n \geq m-1: S_{n} \leq\left(n^{*}\right)^{-1} n^{2} l(n)\right\}
$$

Then we have

$$
N \stackrel{d}{=} Q+1,
$$


which means that the distribution of $N$ is the same as that of $Q+1$. Let

$$
\begin{gathered}
R_{w}=c Q^{2} l(Q)-S_{Q}, c=\left(n^{*}\right)^{-1} \\
r_{c}=c Q^{2}(l(Q)-1)-R_{w} \text { and } Q^{*}=c^{1 / 2}\left(Q-n^{*}\right) .
\end{gathered}
$$

Throughout this section, $M$ denotes a generic positive constant which is independent of $w$. From (2.5), the definition of $Q$ and the results of Woodroofe (1977) we have the following lemma.

LEMMA 3.1. The following results hold.

(i) $Q \stackrel{a . s .}{\rightarrow} \infty, c Q \stackrel{a . s .}{\rightarrow} 1$ as $w \rightarrow 0$ and $c Q^{2}-Q=S_{Q}-Q-r_{c}$, where $\stackrel{\text {, }}{\rightarrow}$ ' denotes almost sure convergence.

(ii) For any fixed $s>0, E\left\{(c Q)^{s}\right\}=O(1)$ and $E\left\{\left(R_{w}\right)^{s}\right\}=O(1)$ as $w \rightarrow 0$.

(iii) $R_{w} \stackrel{d}{\rightarrow} H$ and $r_{c} \stackrel{d}{\rightarrow} l_{0}-H$ as $w \rightarrow 0$

where $H$ is the random variable given in Theorem 2.1 of Woodroofe (1977), $\nu \equiv E(H)=1-\sum_{n=1}^{\infty} n^{-1} E\left\{\left(S_{n}-2 n\right)^{+}\right\}$and $\stackrel{d}{\rightarrow}$ 'stands for convergence in distribution.

(iv) $\sup _{0<w} E|Q(l(Q)-1)|^{s} \leq M \quad$ for any fixed $s>0$.

(v) For any fixed $\beta>0, \quad \sup _{0<w<w_{0}} E\left|r_{c}\right|^{\beta} \leq M$ for some $w_{0}>0$.

(vi) $Q^{*} \stackrel{d}{\rightarrow} N(0,1)$ as $w \rightarrow 0$ where $N(0,1)$ stands for a standard normal random variable.

(vii) For any fixed $s>0,\left\{\left|Q^{*}\right|^{s} ; 0<w<w_{0}\right\}$ is uniformly integrable for $m>\frac{1}{2} s$.

(viii) $Q^{*}$ and $R_{w}$ are asymptotically independent.

(ix) $E(Q)=n^{*}+\nu-l_{0}-1+o(1)$ as $w \rightarrow 0$ for $m>1$.

(x) For any fixed $s>0, E\left\{(c Q)^{-s}\right\}=O(1)$ as $w \rightarrow 0$ for $m \geq s+1$.

By Theorem 2 of Chow, Hsiung and Lai (1979) we can get the following lemma.

LEMMA 3.2. For any fixed $s>0,\left\{\left|c^{1 / 2}\left(S_{Q}-Q\right)\right|^{s} ; 0<w<w_{0}\right\}$ is uniformly integrable for some $w_{0}>0$.

LEMMA 3.3. For any $x>0$ and $n$ with $P(N=n)>0$,

$$
P\left\{\hat{\sigma}_{n} \leq x \mid N=n\right\}=P\left\{\sigma \bar{W}_{n-1} \leq x \mid Q=n-1\right\} .
$$


Proof. Due to Lombard and Swanepoel (1978), $Y \equiv\left\{\sum_{i=2}^{n} Y_{i n}: n \geq 2\right\}$ has the same distribution as $Z=\left\{\sum_{i=1}^{n-1} Z_{i}: n \geq 2\right\}$ where $Y_{i n}$ and $Z_{i}$ are defined at the beginning of this section. The stopping rule $N$ in (2.4) can be rewritten as

$$
N=\inf \left\{n \geq m: \sum_{i=2}^{n} Y_{i n} \leq w(n-1)^{2} l(n-1)\right\} .
$$

Thus from (2.3), (3.1) and (3.2) we have

$$
\begin{aligned}
& P\left\{\hat{\sigma}_{n} \leq x \mid N=n\right\}=P\left\{\hat{\sigma}_{n} \leq x, N=n\right\} / P(N=n) \\
& =P\left\{(n-1)^{-1} \sum_{i=2}^{n} Y_{i n} \leq x, \sum_{i=2}^{k} Y_{i k}>w(k-1)^{2} l(k-1) \text { for } k=2, \ldots, n-1,\right. \\
& \left.\quad \sum_{i=2}^{n} Y_{i n} \leq w(n-1)^{2} l(n-1)\right\} / P(Q=n-1) \\
& =P\left\{(n-1)^{-1} \sum_{i=1}^{n-1} Z_{i} \leq x, \sum_{i=1}^{k-1} Z_{i}>w(k-1)^{2} l(k-1) \text { for } k=2, \ldots, n-1,\right. \\
& \left.\quad \sum_{i=1}^{n-1} Z_{i} \leq w(n-1)^{2} l(n-1)\right\} / P(Q=n-1) \\
& =P\left\{\sigma \bar{W}_{n-1} \leq x, S_{k}>\left(n^{*}\right)^{-1} k^{2} l(k) \text { for } k=1, \ldots, n-2, S_{n-1} \leq\left(n^{*}\right)^{-1}(n-1)^{2}\right. \\
& \quad l(n-1)\} / P(Q=n-1) \\
& =P\left\{\sigma \bar{W}_{n-1} \leq x, Q=n-1\right\} / P(Q=n-1) \\
& =P\left\{\sigma \bar{W}_{Q} \leq x \mid Q=n-1\right\},
\end{aligned}
$$

which concludes the lemma.

LEMMA 3.4. For any fixed $\beta>0,\left\{\left|\left(S_{Q}-Q\right) / Q^{1 / 2}\right|^{\beta} ; 0<w<w_{0}\right\}$ is uniformly integrable for some $w_{0}>0$ if $m>\frac{1}{2} \beta+1$.

PROOF. Choose $\alpha>1$ and $p>1$ such that $m \geq \frac{1}{2} \alpha p \beta+1$. Let $q=p /(p-1)$. Then by Hölder's inequality and Lemmas 3.1 and 3.2 we have

$$
\begin{aligned}
& E\left(\left|\left(S_{Q}-Q\right) / Q^{1 / 2}\right|^{\beta}\right)^{\alpha}=E\left|(c Q)^{-1 / 2} c^{1 / 2}\left(S_{Q}-Q\right)\right|^{\alpha \beta} \\
& \leq\left(E|c Q|^{-\alpha p \beta / 2}\right)^{1 / p}\left(E\left|c^{1 / 2}\left(S_{Q}-Q\right)\right|^{\alpha \beta q}\right)^{1 / q} \leq M
\end{aligned}
$$

for all $0<w<w_{0}$, which gives the uniform integrability of $\left\{\left|\left(S_{Q}-Q\right) / Q^{1 / 2}\right|^{\beta} ; 0<w<\right.$ $\left.w_{0}\right\}$. This completes the proof. 


\section{Proof of Theorem 2.1}

(i) is an immediate consequence of (3.2) and Lemma 3.1. We shall prove (ii). From $(2.3),(3.2)$ and Lemma 3.3 we get

$$
\begin{aligned}
R(\hat{\sigma}, N) & =\sigma^{-1} E\left(\hat{\sigma}_{N}-\sigma\right)^{2}=\sigma^{-1} E\left(\sigma \bar{W}_{Q}-\sigma\right)^{2} \\
& =w E\left\{\left(n^{*} / Q^{2}\right)\left(S_{Q}-Q\right)^{2}\right\} \\
& =w\left[E\left\{\left(S_{Q}-Q\right)^{2} / n^{*}\right\}+n^{*} E\left\{\left(Q^{-2}-n^{*-2}\right)\left(S_{Q}-Q\right)^{2}\right\}\right],
\end{aligned}
$$

which, together with Theorem 2 of Chow, Robbins and Teicher (1965) and Lemma 3.1, implies

$$
E\left(S_{Q}-Q\right)^{2}=E(Q)=n^{*}+\nu-l_{0}-1+o(1) \text { as } w \rightarrow 0
$$

Hence

$$
\begin{aligned}
R(\hat{\sigma}, N) / w=1 & +\left(\nu-l_{0}-1\right) / n^{*}+o\left(1 / n^{*}\right) \\
& +\left(n^{*}\right)^{-1} E\left\{(c Q)^{-2}(1+c Q)(1-c Q)\left(S_{Q}-Q\right)^{2}\right\} .
\end{aligned}
$$

Set

$$
I=(c Q)^{-2}(1+c Q)(1-c Q)\left(S_{Q}-Q\right)^{2}
$$

Then

$$
n^{*}\{R(\hat{\sigma}, N) / w-1\}=\nu-l_{0}-1+E(I)+o(1) \text { as } w \rightarrow 0 .
$$

Since by Lemma 3.1 (i)

$$
1-c Q=-\left(S_{Q}-Q\right) Q^{-1}+r_{c} Q^{-1}
$$

we get from (3.3) that

$$
\begin{aligned}
I & =-(c Q)^{-2}(1+c Q)\left(S_{Q}-Q\right)^{3} Q^{-1}+(c Q)^{-2}(1+c Q) r_{c}\left(S_{Q}-Q\right)^{2} Q^{-1} \\
& \equiv-I_{1}+I_{2}, \text { say. }
\end{aligned}
$$

In order to prove (ii), it is sufficient from (3.4) and (3.6) to show

$$
E\left(I_{1}\right)=-5+o(1) \text { as } w \rightarrow 0
$$

and

$$
E\left(I_{2}\right)=2 l_{0}-2 \nu+o(1) \text { as } w \rightarrow 0 .
$$

We shall first prove (3.7). It follows from (3.5) that 


$$
\begin{aligned}
I_{1} & =(c Q)^{-2}\left\{2 c\left(S_{Q}-Q\right)^{3}+(1-c Q)\left(S_{Q}-Q\right)^{3} / Q\right\} \\
& =2(c Q)^{-2} c\left(S_{Q}-Q\right)^{3}-(c Q)^{-2}\left(S_{Q}-Q\right)^{4} / Q^{2}+(c Q)^{-2} r_{c}\left(S_{Q}-Q\right)^{3} / Q^{2} \\
& \equiv 2 I_{11}-I_{12}+I_{13}, \text { say. }
\end{aligned}
$$

By Lemma 3.1 (i) and the result of Anscombe (1952) we get $\left(S_{Q}-Q\right)^{4} / Q^{2} \stackrel{d}{\rightarrow} \chi_{1}^{4}$ as $w \rightarrow$ 0 , where $\chi_{1}^{2}$ denotes a chi-squared random variable with one degree of freedom. Hence it follows from Lemma 3.1 that

$$
I_{12} \stackrel{d}{\rightarrow} \chi_{1}^{4} \text { and } I_{13} \stackrel{p}{\rightarrow} 0 \text { as } w \rightarrow 0
$$

where ' $\stackrel{p}{\rightarrow}$ ' stands for convergence in probability. Since $m>5$, we can choose constants $\alpha>1$ and $p>1$ such that $m \geq 4 \alpha p+1$. Let $q=p /(p-1)$. Then by Hölder's inequality and Lemmas 3.1 and 3.2 we get

$$
\begin{aligned}
E\left|I_{12}\right|^{\alpha} & =E\left|(c Q)^{-4}\left\{c^{1 / 2}\left(S_{Q}-Q\right)\right\}^{4}\right|^{\alpha} \\
& \leq\left(E(c Q)^{-4 \alpha p}\right)^{1 / p}\left(E\left|c^{1 / 2}\left(S_{Q}-Q\right)\right|^{4 \alpha q}\right)^{1 / q} \leq M
\end{aligned}
$$

for all $0<w<w_{0}$. This yields the uniform integrability of $\left\{I_{12} ; 0<w<w_{0}\right\}$. Thus from $(3.10)$

$$
E\left(I_{12}\right)=3+o(1) \text { as } w \rightarrow 0 .
$$

Throughout this section we use the above method to prove uniform integrabilities of sequences of random variables, and omit their proofs because the calculations are tedious. Since $I_{13}=\left\{c^{1 / 2}\left(S_{Q}-Q\right)\right\}^{3} r_{c}(c Q)^{-7 / 2} Q^{-1 / 2}$, it follows from Lemmas 3.1 and 3.2 that $\left\{I_{13} ; 0<w<w_{0}\right\}$ is uniformly integrable. Thus by $(3.10)$ we get

$$
E\left(I_{13}\right)=o(1) \text { as } w \rightarrow 0,
$$

which, together with (3.9) and (3.11), yields

$$
E\left(I_{1}\right)=2 E\left(I_{11}\right)-3+o(1) \text { as } w \rightarrow 0 .
$$

Set

$$
J_{1}=\left\{(c Q)^{-2}-1\right\} c\left(S_{Q}-Q\right)^{3} \text { and } J_{2}=\left(S_{Q}-Q\right)^{3}
$$

Then

$$
E\left(I_{11}\right)=E\left(J_{1}\right)+c E\left(J_{2}\right) .
$$

From Theorem 9 of Chow et al. (1965) and Lemma 3.1 (ix) we have

$$
E\left(J_{2}\right)=2 c^{-1}+2\left(\nu-l_{0}-1\right)+3 E\left\{Q\left(S_{Q}-Q\right)\right\}+o(1)
$$


By (3.5) and Wald's equation we get

$$
\begin{aligned}
E\left\{Q\left(S_{Q}-Q\right)\right\} & =c^{-1} E\left\{(c Q)\left(S_{Q}-Q\right)\right\} \\
& =c^{-1}\left[E\left(S_{Q}-Q\right)+E\left\{\left(S_{Q}-Q\right)^{2} / Q\right\}-E\left\{r_{c}\left(S_{Q}-Q\right) / Q\right\}\right] \\
& =c^{-1}\left[E\left\{\left(S_{Q}-Q\right)^{2} / Q\right\}-E\left\{r_{c}\left(S_{Q}-Q\right) / Q\right\}\right] .
\end{aligned}
$$

It follows from Lemma 3.4 that $\left\{\left(S_{Q}-Q\right)^{2} / Q ; 0<w<w_{0}\right\}$ is uniformly integrable. Hence by the fact that $\left(S_{Q}-Q\right)^{2} / Q \stackrel{d}{\rightarrow} \chi_{1}^{2}$ as $w \rightarrow 0$, we have

$$
E\left\{\left(S_{Q}-Q\right)^{2} / Q\right\}=1+o(1) \text { as } w \rightarrow 0 .
$$

By using Lemma 3.1 and the strong law of large numbers (SLLN), we can show that $r_{c}\left(S_{Q}-Q\right) / Q \stackrel{p}{\rightarrow} 0$ as $w \rightarrow 0$ and that $\left\{r_{c}\left(S_{Q}-Q\right) / Q ; 0<w<w_{0}\right\}$ is uniformly integrable, which yield

$$
E\left\{r_{c}\left(S_{Q}-Q\right) / Q\right\}=o(1) \text { as } w \rightarrow 0 .
$$

Thus (3.16) and (3.17) give $E\left\{Q\left(S_{Q}-Q\right)\right\}=c^{-1}(1+o(1))$ as $w \rightarrow 0$, which, together with (3.14) and (3.15), implies

$$
E\left(I_{11}\right)=E\left(J_{1}\right)+5+o(1) \text { as } w \rightarrow 0 .
$$

Since $\left\{(c Q)^{-2}-1\right\} c=-\left\{(c Q)^{-1}+1\right\} c\left(Q-n^{*}\right) / Q$ and $c\left(Q-n^{*}\right)=\left(S_{Q}-Q\right) / Q-r_{c} / Q$ by Lemma 3.1 (i), we get from (3.13) that

$$
\begin{aligned}
J_{1} & =-\left\{(c Q)^{-1}+1\right\}\left(S_{Q}-Q\right)^{4} / Q^{2}+\left\{(c Q)^{-1}+1\right\} r_{c}\left(S_{Q}-Q\right)^{3} / Q^{2} \\
& \equiv-J_{11}+J_{12}, \text { say. }
\end{aligned}
$$

From Lemma 3.1 and the fact that $\left(S_{Q}-Q\right) / Q^{1 / 2} \stackrel{d}{\rightarrow} N(0,1)$ as $w \rightarrow 0$, we have

$$
J_{11} \stackrel{d}{\rightarrow} 2 \chi_{1}^{4} \text { and } J_{12} \stackrel{p}{\rightarrow} 0 \text { as } w \rightarrow 0 .
$$

By Lemmas 3.1 and 3.4 we can show the uniform integrability of $\left\{J_{11} ; 0<w<w_{0}\right\}$, which, together with $(3.20)$, yields

$$
E\left(J_{11}\right)=6+o(1) \text { as } w \rightarrow 0 .
$$

By using (3.20) and the uniform integrability of $\left\{J_{12} ; 0<w<w_{0}\right\}$ we have $E\left(J_{12}\right)=$ $o(1)$ as $w \rightarrow 0$, which, together with (3.19) and (3.21), implies $E\left(J_{1}\right)=-6+$ $o(1)$ as $w \rightarrow 0$. Thus from (3.12) and (3.18) we get $E\left(I_{1}\right)=-5+o(1)$ as $w \rightarrow 0$, which gives (3.7). Next we shall show (3.8). Lemma 3.1 (i) implies

$$
(c Q)^{-2}\left(S_{Q}-Q\right)^{2}=c^{-1} Q^{* 2}+2(c Q)^{-1}\left(Q-n^{*}\right) r_{c}+(c Q)^{-2} r_{c}^{2} .
$$


Hence from (3.6)

$$
\begin{aligned}
I_{2} & =\left\{1+(c Q)^{-1}\right\} r_{c} Q^{* 2}+2\left\{1+(c Q)^{-1}\right\} r_{c}^{2}\left(Q-n^{*}\right) / Q+(1+c Q)(c Q)^{-2} r_{c}^{3} Q^{-1} \\
& \equiv I_{21}+I_{22}+I_{23}, \text { say. }
\end{aligned}
$$

From Lemma 3.1 we have

$$
I_{22} \stackrel{p}{\rightarrow} 0 \text { and } I_{23} \stackrel{p}{\rightarrow} 0 \text { as } w \rightarrow 0 .
$$

According to the fact that for $\alpha>1,\left|I_{22}\right|^{\alpha} \leq M\left|(c Q)^{-\frac{1}{2}} Q^{*} r_{c}{ }^{2}\right|^{\alpha}+\left|(c Q)^{-\frac{3}{2}} Q^{*} r_{c}{ }^{2}\right|^{\alpha}$ and Lemma 3.1, we obtain the uniform integrability of $\left\{I_{22} ; 0<w<w_{0}\right\}$, which, together with (3.23), implies

$$
E\left(I_{22}\right)=o(1) \text { as } w \rightarrow 0 .
$$

By using the fact that $Q^{-1} \leq 1$ we get the uniform integrability of $\left\{I_{23} ; 0<w<w_{0}\right\}$, which, together with (3.23), yields

$$
E\left(I_{23}\right)=o(1) \text { as } w \rightarrow 0 .
$$

Hence from (3.22) and (3.24) we get

$$
E\left(I_{2}\right)=E\left(I_{21}\right)+o(1) \text { as } w \rightarrow 0 .
$$

Since $r_{c}=c Q^{2}(l(Q)-1)-R_{w}$,

$$
\begin{aligned}
I_{21} & =\left\{1+(c Q)^{-1}\right\} Q^{* 2} c Q^{2}(l(Q)-1)-\left\{1+(c Q)^{-1}\right\} Q^{* 2} R_{w} \\
& \equiv I_{211}-I_{212}, \text { say. }
\end{aligned}
$$

Let $K$ be a standard normal random variable which is independent of $H$. Then by Lemma 3.1 (iii), (vi) and (viii) we have

$$
\left(Q^{*}, R_{w}\right) \stackrel{d}{\rightarrow}(K, H) \text { and } c Q^{2}(l(Q)-1) \stackrel{a \cdot s .}{\rightarrow} l_{0} \text { as } w \rightarrow 0
$$

which imply

$$
I_{211} \stackrel{d}{\rightarrow} 2 l_{0} K^{2} \text { and } I_{212} \stackrel{d}{\rightarrow} 2 K^{2} H \quad \text { as } w \rightarrow 0 .
$$

From Lemma 3.1 we can show the uniform integrability of $\left\{I_{211} ; 0<w<w_{0}\right\}$, which yields

$$
E\left(I_{211}\right)=2 l_{0}+o(1) \text { as } w \rightarrow 0 .
$$

By the independency of $K^{2}$ and $H$ we have

$$
E\left(K^{2} H\right)=E\left(K^{2}\right) E(H)=\nu
$$


Since we can show the unifom integrability of $\left\{I_{212} ; 0<w<w_{0}\right\}$, we have by (3.27) and (3.29) that

$$
E\left(I_{212}\right)=2 \nu+o(1) \text { as } w \rightarrow 0
$$

which, together with $(3.25),(3.26)$ and $(3.28)$, implies

$$
E\left(I_{2}\right)=2 l_{0}-2 \nu+o(1) \text { as } w \rightarrow 0 .
$$

Thus (3.8) is shown. Therefore the proof of Theorem 2.1 is complete.

\section{Proof of Proposition 2.1.}

From (2.3), (3.2), Lemma 3.3 and Wald's equation we have

$$
\begin{aligned}
E\left(\hat{\sigma}_{N}\right)-\sigma & =\sum_{n=m}^{\infty} \int(x-\sigma) d P\left(\hat{\sigma}_{n} \leq x \mid N=n\right) P(N=n) \\
& =\sum_{n=m}^{\infty} \int(x-\sigma) d P\left(\sigma \bar{W}_{n-1} \leq x \mid Q=n-1\right) P(Q=n-1) \\
& =E\left(\sigma \bar{W}_{Q}-\sigma\right) \\
& =w E\left(S_{Q}-Q\right)+w n^{*} E\left\{\left(Q^{-1}-n^{*-1}\right)\left(S_{Q}-Q\right)\right\} \\
& =w E\left\{(c Q)^{-1}(1-c Q)\left(S_{Q}-Q\right)\right\} \\
& \equiv w E(I), \text { say. }
\end{aligned}
$$

By (3.5), Lemma 3.1 and SLLN we get

$$
\begin{aligned}
& I=-(c Q)^{-1}\left(S_{Q}-Q\right)^{2} / Q+(c Q)^{-1} r_{c}\left(S_{Q}-Q\right) / Q \\
& \stackrel{d}{\rightarrow}-\chi_{1}^{2} \text { as } w \rightarrow 0 .
\end{aligned}
$$

Since $I=-(c Q)^{-1} Q^{*} c^{1 / 2}\left(S_{Q}-Q\right)$ it follows from Lemmas 3.1 and 3.2 that $\{I ; 0<$ $\left.w<w_{0}\right\}$ is uniformly integrable. Thus (3.31) implies that $E(I)=-1+o(1)$ as $w \rightarrow 0$, which, together with $(3.30)$, concludes the proposition. Therefore, the proof is complete.

\section{Proof of Theorem 2.2}

From (2.6) we get

$$
R\left(\hat{\sigma}^{*}, N\right)=R(\hat{\sigma}, N)+2 w \sigma^{-1} E\left(\hat{\sigma}_{N}-\sigma\right)+w^{2} \sigma^{-1} .
$$

Thus, by using (2.3), Theorem 2.1 and Proposition 2.1 we obtain Theorem 2.2. This completes the proof. 


\section{Acknowledgements}

The first author was partially supported by a Grant-in-Aid for Science Research, number 09640251, from the Ministry of Education, Science and Culture of Japan. We would like to thank the referee for his helpful comments.

\section{References}

Anscombe, F.J. (1952), Large sample theory of sequential estimation, Proc. Cambridge Philos. Soc., 48, 600-607.

Chow, Y.S., Hsiung, C. and Lai, T.L. (1979), Extended renewal theory and moment convergence in Anscombe's theorem, Ann. Probab., 7, 304-318.

Chow, Y.S., Robbins, H. and Teicher, H. (1965), Moments of randomly stopped sums, Ann. Math. Statist., 36, 789-799.

Ghosh, M., Mukhopadhyay, N. and Sen, P. K. (1997), Sequential Estimation, Wiley, New York.

Lombard, F. and Swanepoel, J.W.H. (1978), On finite and infinite confidence sequences, South African Statist. J., 12, 1-24.

Mukhopadhyay, N. (1988), Sequential estimation problems for negative exponential populations, Commun. Statist. Theor. Math., 17(8), 2471-2506.

Woodroofe, M. (1977), Second order approximations for sequential point and interval estimation, Ann. Statist., 5, 984-995.

Received July 16, 1998

Revised October 19, 1998 